\title{
Design and implementation of UWB systems with timing synchronization in MATLAB Simulink
}

\author{
L.V.Santosh Kumar Y ${ }^{1}$, Y.V Sri Charan ${ }^{2}$, K.H.K Raghu Vamsi ${ }^{3}$ \\ ${ }^{1,2}$ (Assistant Professor, Raghu Engineering College, India) \\ ${ }^{3}$ (Assistant Professor, Aditya Engineering College, India)
}

\begin{abstract}
Ultra wide band (UWB) bandwidth is much higher than the system bandwidth requirement. Due to large bandwidth in UWB, its systems must have time resolution for system time distribution. But we need to improve data rate and efficiency of the system which uses ultra wide band channels as data rate may trade for power spectral density and performance in multipath. In order to increase data rate, here in this project the orthogonal frequency division multiplexing (OFDM) system is used. But we need to overcome the drawbacks in OFDM like peak to average power ratio (PAPR) [5], carrier frequency offset (CFO), inter symbol interference (ISI), inter carrier interference (ICI). In order to control the effect of PAPR and CFE the single carrier with frequency domain equalization (SC-FDE) can be used, as it has lower PAPR and lower sensitivity to CFO compared to OFDM but the problem is that it is less robust to timing error. As the data rate in UWB systems is high, there is heavy requirement of accuracy in timing synchronization constraints. Moreover the high dispersive nature of UWB channels is an extra challenge to acquire timing synchronization. In general, synchronization can be done based on auto correlation and cross correlation methods. Joint timing and channel estimation (JTCE) can also be done but the computational complexity while correlating will be more compared to correlation methods. But, for UWB systems the timing schemes cannot perform well as multi path UWB channels are denser and longer than wideband channels. Due to trade off's and computational complexity the implementation of large size Fast Fourier transform (FFT) and Inverse FFT (IFFT) can't be employed. In this paper, we discuss the design and implementation of UWB systems with timing synchronization by avoiding all the above discussed PAPR, CFO, ISI, ICI and timing synchronization in $U W B$ channel problems and results are compared by transmitting signal through three different channels (dispersive, fading and addictive white Gaussian noise (AWGN) channels).
\end{abstract}

Keywords - CFO, ISI, ICI, JTCE, OFDM, PAPR, SC-FDE, UWB

\section{Introduction}

In General, Ultra wide band (UWB) bandwidth is much higher than the system bandwidth requirement. So Federal Communications Commission (FCC) decided to divide the bandwidth into narrow frequency bands, where more amount of information can be transmitted by different users. Because of that the data rate will be increased but the systems complexity and disadvantages also will increase with advantages. Due to large bandwidth in UWB, its systems must have time resolution for system time distribution. But we need to improve data rate and efficiency of the system which uses ultra wide band channels as data rate may trade for power spectral density and performance in multipath. There are two common types of UWB, Impulse ultra wide band (I-UWB) and Multi carrier wide band (MC-UWB). Sending pulses with short duration to transmit information is I-UWB and MC-UWB is sending information through multiple carriers. In order to have high data rates to a system, MC-UWB is preferred as more information can be transmitted through channel in particular interval time. The most popular multi carrier system is OFDM technology, the system with OFDM modulation can provide high data rates efficiently as multiple orthogonally spaced sub carriers are used as carriers for data transmission. Now OFDM technology is used in Asymmetric Digital Subscriber Line (ADSL) services, digital broadcasting services in Europe. 3G technology emerged based on OFDM technology and it is also considered for 4G technology also. This has lots of literature survey where many issues were discussed in different applications and many design models to control the problems in OFDM but all of them are up to that specific application only. So, we need a specific design which is compatible to every system in UWB range with efficient performance and this paper is about the implementation of design of OFDM system in order to attain such characteristics.

OFDM requires very accurate frequency synchronization between the receiver and the transmitter [1]. With frequency deviation the sub-carriers will no longer be orthogonal, causing inter-carrier interference (ICI) (i.e., crosstalk between the sub-carriers). Frequency offsets are typically caused by mismatched transmitter and receiver oscillators, or by Doppler shift due to movement. So the start of the OFDM frame and the beginning of the symbol 
Design and implementation of UWB systems with timing synchronization in MATLAB Simulink

must be identified and sub channel carrier frequency offsets must be corrected. The paper was completely implemented using MATLAB Simulink. In simulink, we design the transmitter, channel, timing control unit and receiver using blocks which have specified input variables which are to be used. It gives us the physical experience of usage of system though the blocks run on programs at the backend. We can get the sense of output at the end of every block which shows the realizable nature of the designed system.

The organization of the paper is as follows: In section I the introduction to UWB communications, purpose of OFDM in UWB and the simulation tool to implement the system design was discussed. In section II, OFDM transmitter implementation including with internal blocks was discussed and also the implementation of the design using simulink. In section III, including with implementation in simulink, the design and analysis part of OFDM receiver is discussed in detail. In section IV purpose and significance of timing control unit in system's operation is discussed and also implementation in simulation tool. In section $\mathrm{V}$ shows the simulation results and finally in section VI the paper was concluded based on the results in section V.

\section{OFDM Transmitter}

Transmitter in OFDM includes modulator, serial to parallel convertor, IFFT, guard interval insertion/ cyclic prefix insertion and parallel to serial convertor. In this paper, the inputs to the OFDM transmitter block are variable symbol timing (VST) and carrier frequency error (CFE) [1]. Where, the variable symbol timing is used to detect the starting point of each and every OFDM symbol after removing cyclic prefix, which provides exact samples. In order to avoid inter carrier interference the carrier frequency error is introduced and the implementation in Simulink is shown in figure 1.

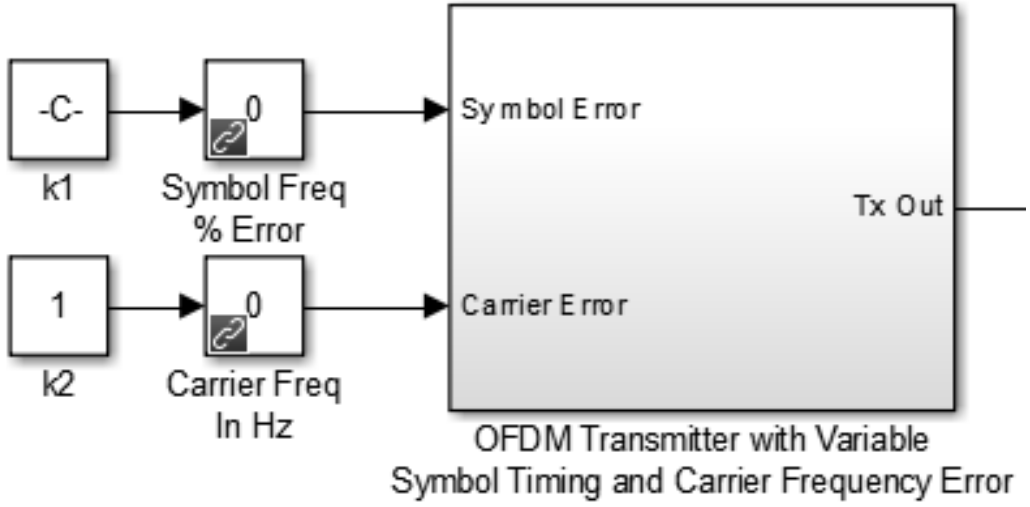

Figure 1: OFDM Transmitter with VST and CFE inputs

In OFDM transmitter with VST and CFE inputs, we actually need to generate OFDM symbols, so with respect to OFDM frame length, the OFDM generator is implemented, where the output samples serially as shown in figure 2 .

Figure 3 shows the internal structure of OFDM generator which consists of random binary bit generator, modulator, and transmitter. Bernoulli random generator generates binary bits randomly, and then the input data is modulated using rectangular quadrature amplitude modulator, where M-QAM (M array Quadrature Amplitude Modulation) is used in modulator, $\mathrm{M}$ represents number of symbols which is equal $2^{\mathrm{N}}$, where $\mathrm{N}$ represents number of bits. Here rectangular 4-QAM is used as modulator. The output of the modulator is given to OFDM transmitter which includes serial to parallel convertor, Inverse FFT and cyclic prefix as shown in figure 4. IFFT is considered as OFDM modulator where the frequency mapping will be done with orthogonal sub-carriers. In order to avoid ISI, cyclic prefix is included. In cyclic prefix, last bit of OFDM frame obtained after IFFT is added to the beginning of the OFDM frame which gives the complete OFDM frame that is to be transmitted. The OFDM frame is converted to serial frame by using frame conversion as shown in figure 3. 


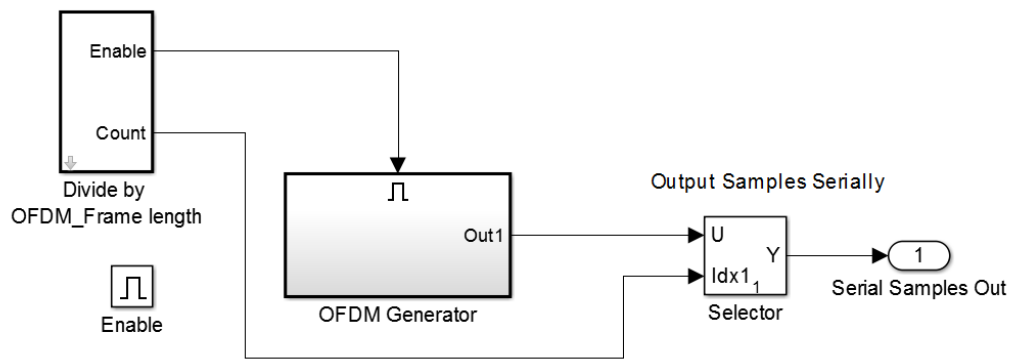

Figure 2: OFDM Transmitter internal structure with frame length divider

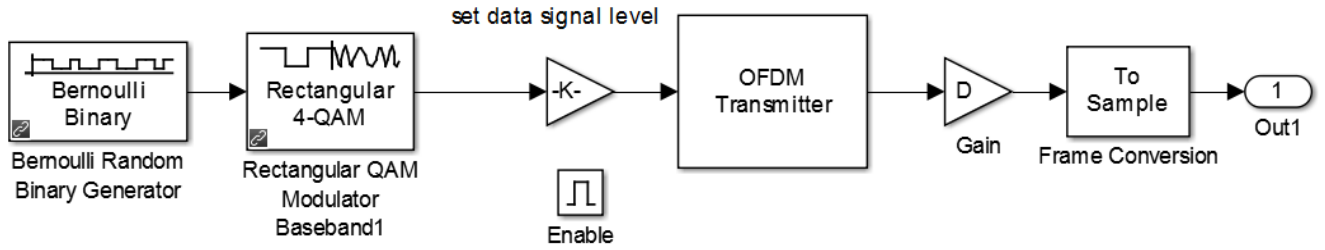

Figure 3: OFDM Generator

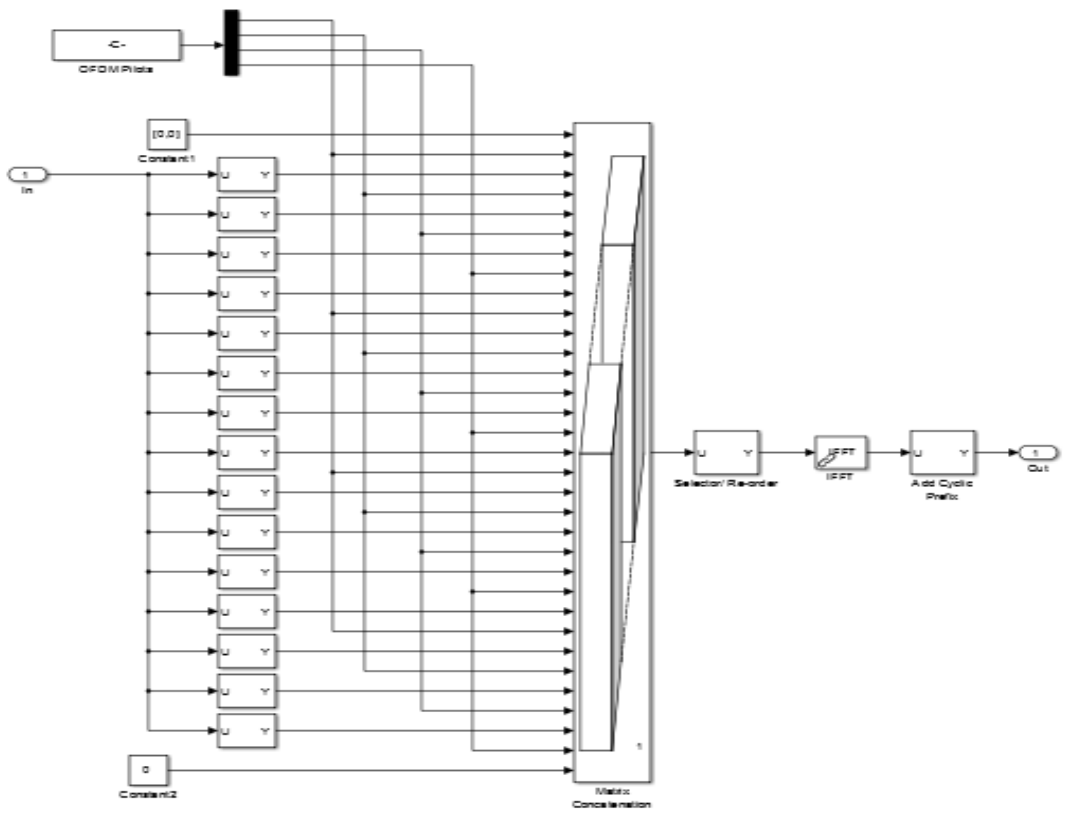

Figure 4: Internal structure of OFDM Transmitter

\section{OFDM Receiver}

The ODFM modulated signal is transmitted through channel and the received OFDM signal is given to the OFDM receiver. The OFDM receiver block consists of cyclic prefix remover, FFT, parallel to serial convertor as shown in the figure 5. Initially the received ODFM frame is applied to cyclic prefix remover, where it removes the extra bit that was arranged in the transmitter side.

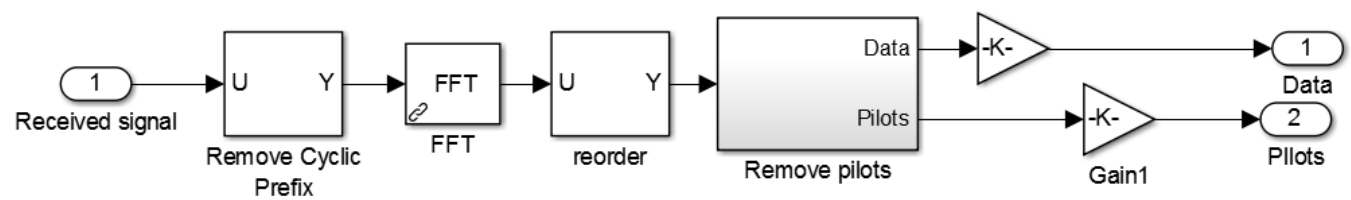

Figure 5: OFDM receiver 
Then, the data is given to FFT in order to perform OFDM demodulation that is frequency de-mapping is done. Then finally the data is rearranged in order and then the pilot carriers are removed by selecting the specified elements of input signal by identifying its element's index from input port. In OFDM demodulated signal, data and pilots are separated and processed separately as shown in figure 6. Channel coefficients are estimated from impulse response of the system, estimated frame offset and channel magnitude are obtained from channels A and B respectively [3]. From the data, the constellation plot of data is figured out, in order to know the interference in the received bits and also bit error rate (BER) [6] is calculated from the same.

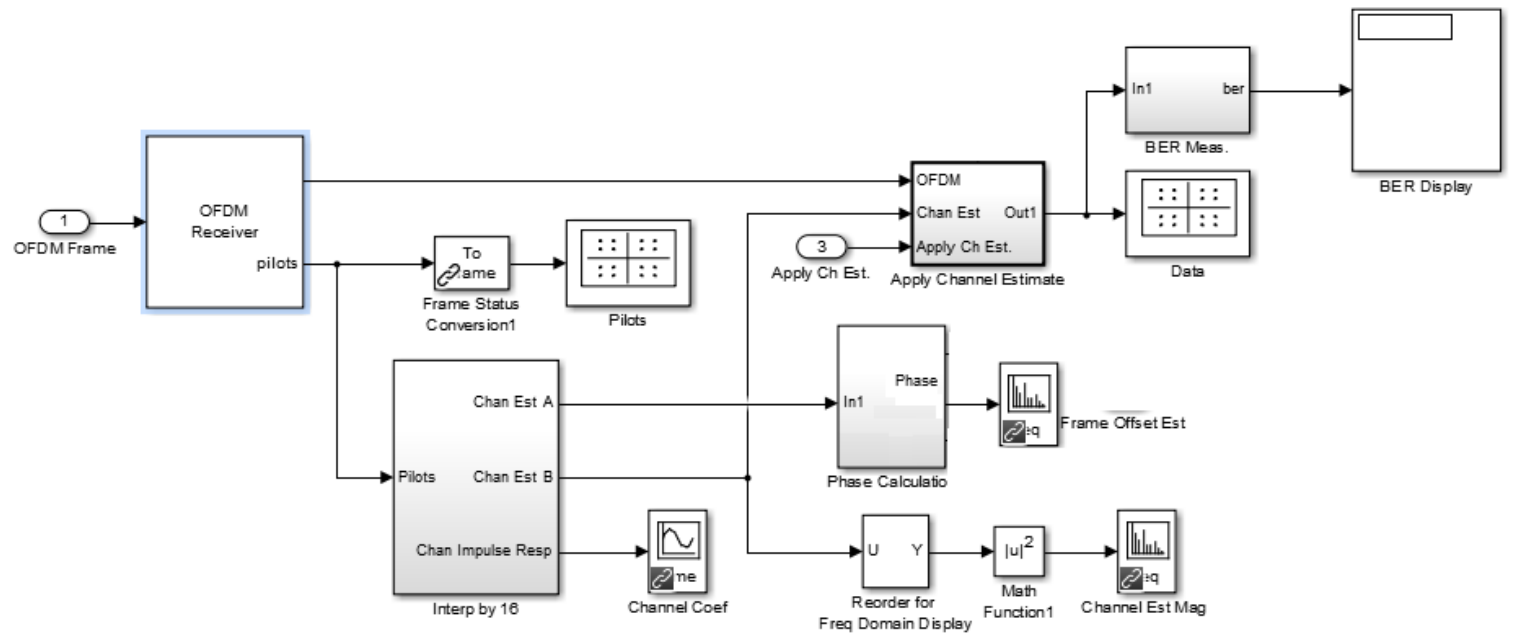

Figure 6: Calculation of BER, Estimated channel magnitude and estimated frame offset

\section{Timing Control Unit}

In order to perform all the operations in receiver, the delay must be controlled; the processing must be enabled only when the timing synchronization is done. The timing control unit is shown in figure 7 . The delay elements are controlled by the math function and relational operator. The conditional value will be verified by relational operator and the result is considered as one of the output named 'strobe', the other output 'delay control' is taken by adjusting phase, and the last output shows the 'error in percentage' which gives normalized error value [4]. The complete block diagram of OFDM that was designed in MATLAB Simulink is shown in figure 8.

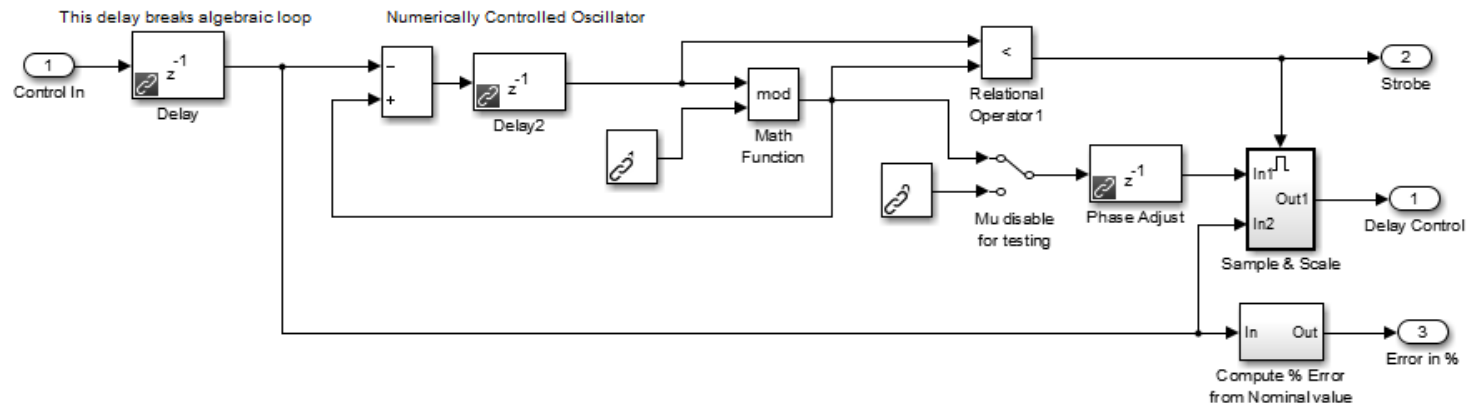

Figure 7: Timing control unit 

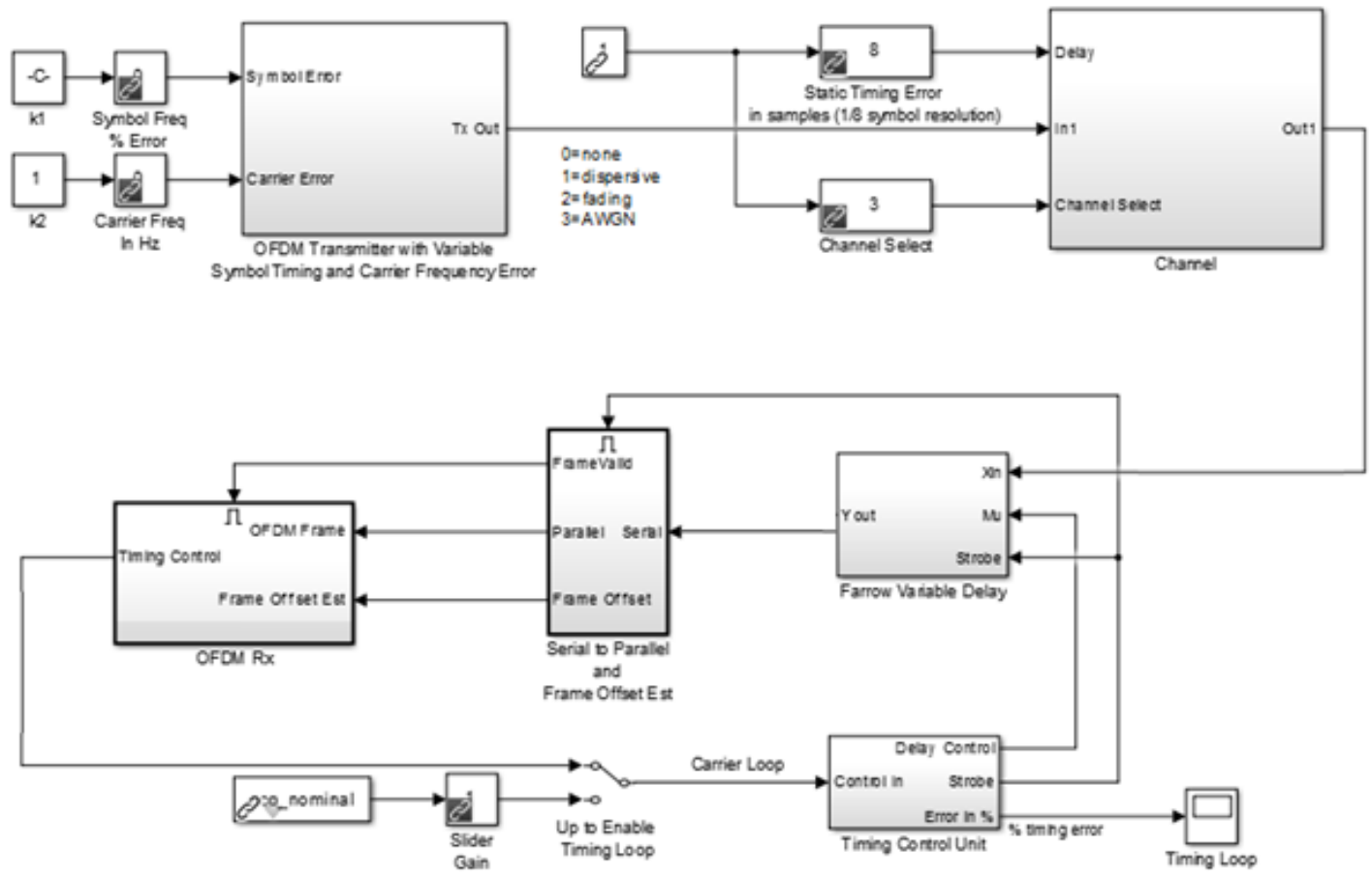

Figure 8: Complete block diagram OFDM for UWB communications

\section{Simulation Results}

As shown in figure 8 , initially after giving required inputs to the system, we need to select the channel that is here in this project the analysis is done three different channels, namely dispersive channel, fading channel and AWGN channel [2]. The simulation results shown below are obtained when channel is in AWGN channel. Figure 9 shows the graph of amplitude of channel coefficients in receiver with respect to time in nano-seconds. Figure 10 shows the graph of estimated channel magnitude in receiver with respect to frequency in kHz. Figure 11 shows the estimated phase response in receiver with respect frequency in kHz. All the figures 9, 10, 11 give the analysis of received signal in terms of coefficients, magnitude and phase. The response varies as the channel selection varies.

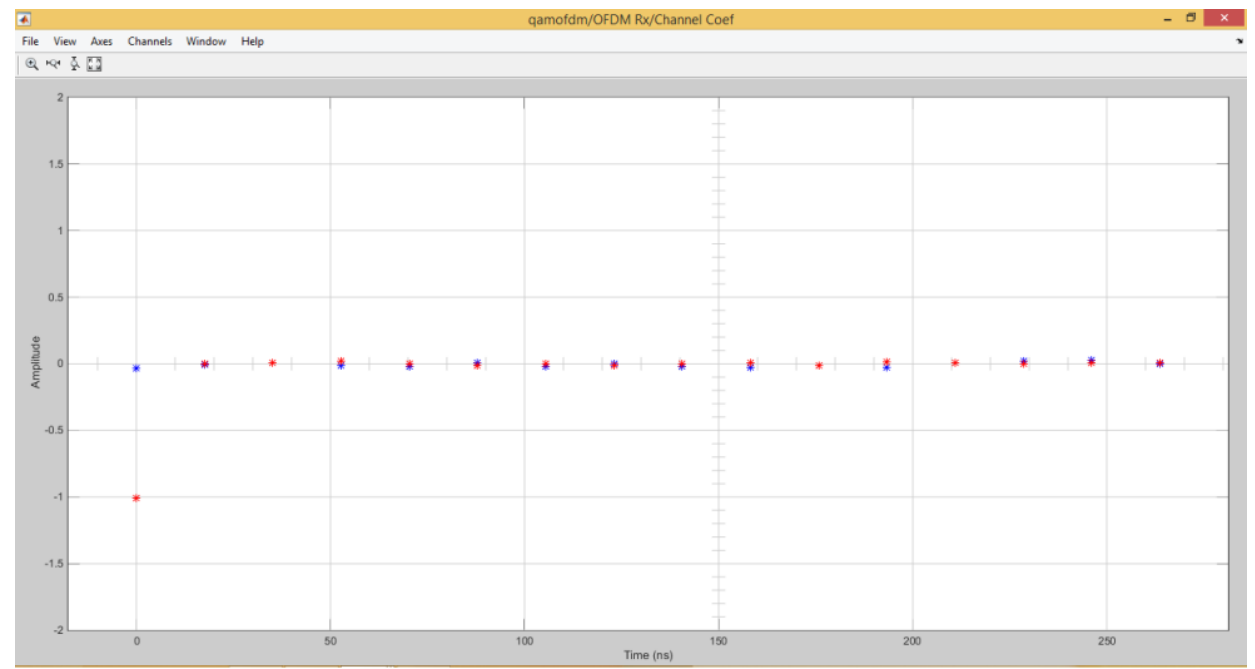

Figure 9: Channel coefficients in receiver 


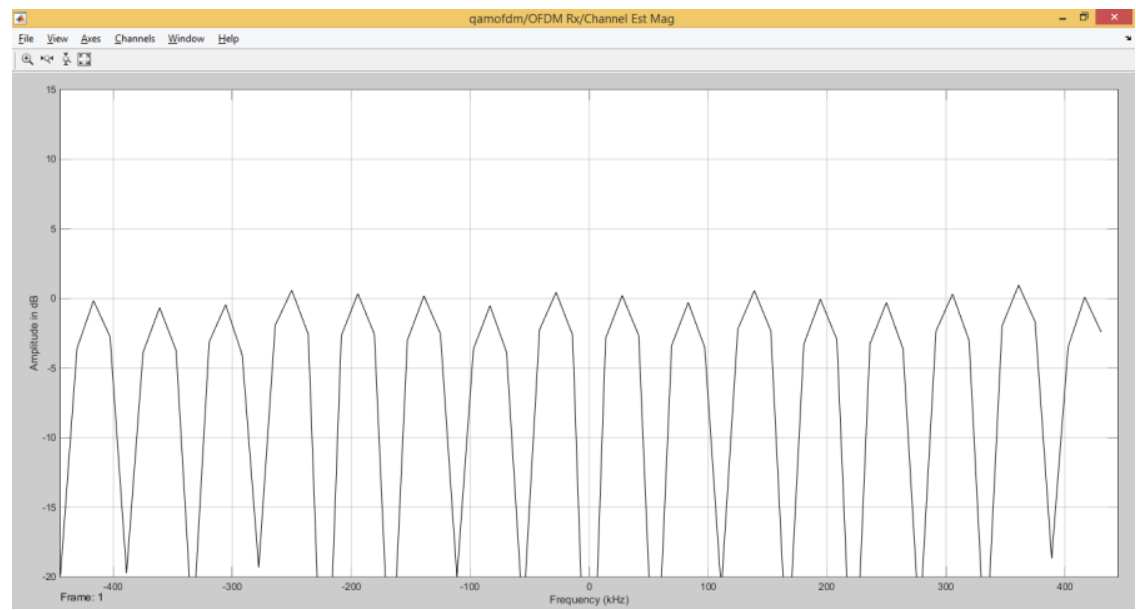

Figure 10: Estimated channel magnitude in receiver

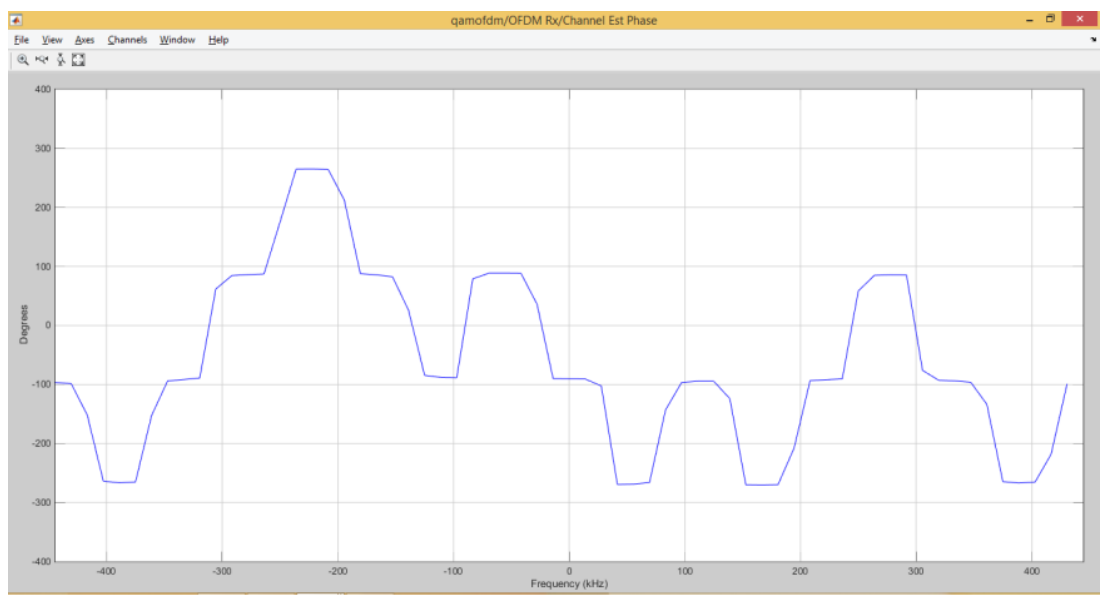

Figure 11: Estimated channel phase in receiver

Figure 12 shows the scattered plot of received data for 4-Quadrature Amplitude Modulation technique and figure 13 shows the scattered plot of pilots at receiver. From the figures 12 and 13 we can understand that the mapping of the data and pilots are very much apart from each other. Both the data and pilots are separated with the phase shift of $90^{\circ}$ and the distant points are far from each other which avoids interference and loss of data. The positions of the data points will be varied continuously as the data is randomly generated from random Bernoulli generator.

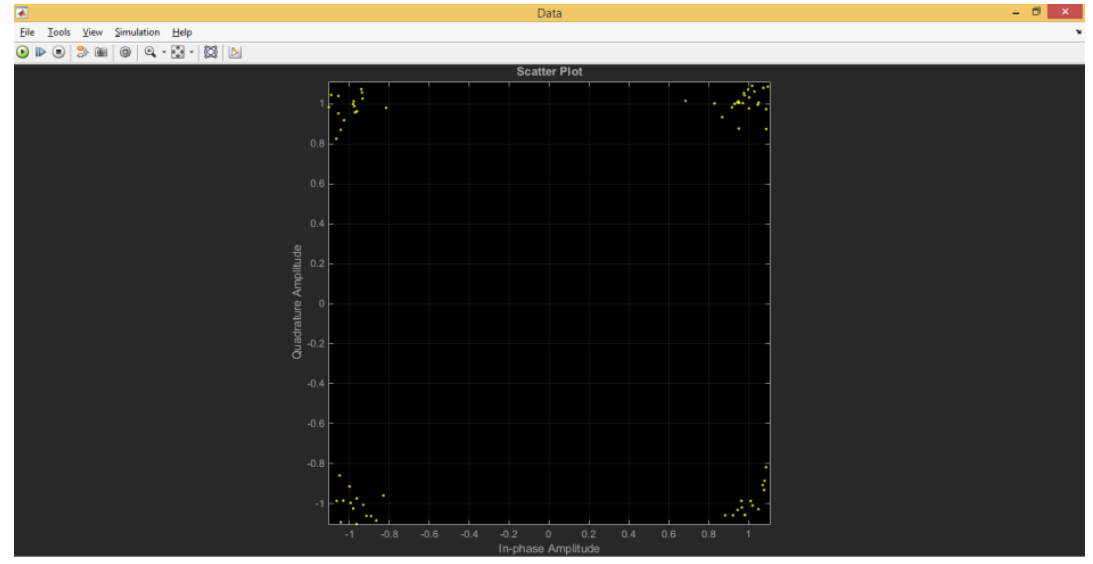

Figure 12: Scattered plot of received data 


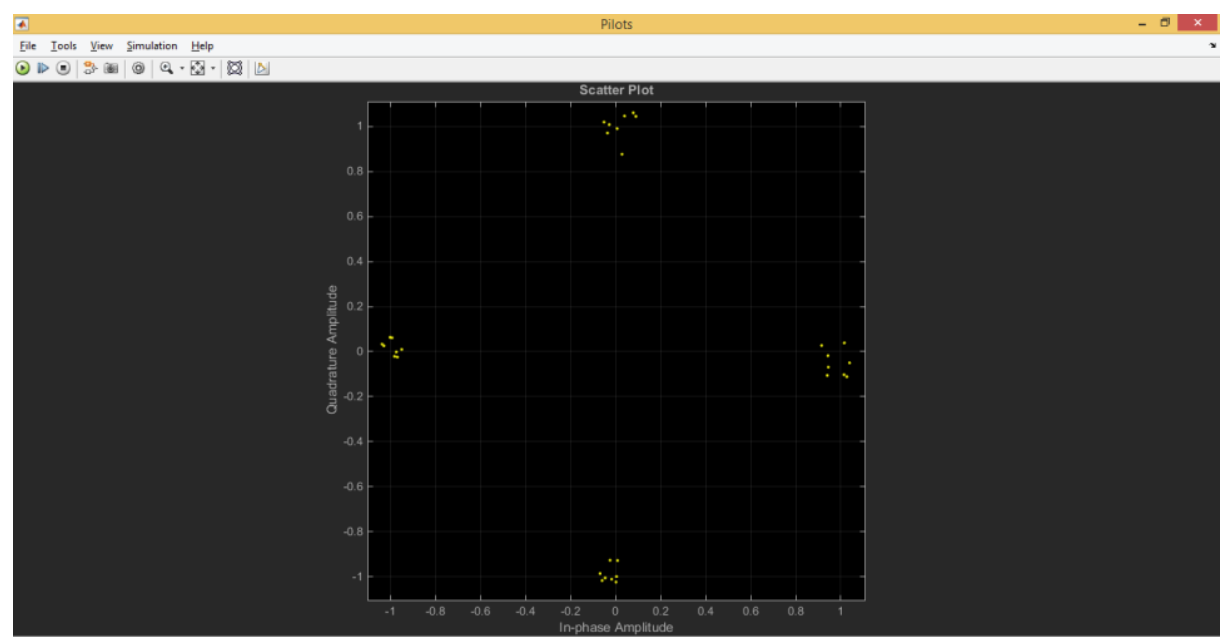

Figure 13: Scattered plots of pilots

\section{Conclusion}

From the obtained simulation results, we would like to conclude that our OFDM design works efficiently in ultra wide band by avoiding the drawbacks in OFDM, including with timing control. As the number inputs are applied randomly continuously the bit error rate is continuously varied. When dispersive and fading type channels are used, the results varies continuously, the scattering plot of data and pilots shows interference of points. As it happens, there will be loss in signal but in AWGN channel the systems shows the stable results, which shows the efficiency of system's design and reliability to the channel. So, our proposed design is best suited for UWB communications in AWGN channel. The future work can be extended if we could work with system design and implementation for combination of channels with further advancements like SC-FDE including with timing control unit to avoid the problems in OFDM.

\section{References}

[1]. MIMO-OFDM wireless communications with MATLAB, Yong Soo Cho, Jaekwon Kim, Won Young Yang, Chung G. Kang, Korea University, (IEEE Press, WILEY)

[2]. STC-Mimo Block Spread OFDM in Frequency Selective AWGN Channels. V Siva Bhaskara Rao, G. Sasibhushan Rao, L V Santosh Kumar Y, International Journal of Research, Vol.3 No. 10

[3]. Tzi-Dar Chiueh, Pei-Yun Tsai, I-Wei Lai, Baseband Receiver Design for Wireless MIMO-OFDM Communications, ( IEEE WILEY II Edition)

[4]. $\quad$ R.W Chang, “Orthogonal Frequency Division Multiplexing,” U.S Patent 3388455, Jan 6, 1970, Filed Nov.4.1966.

[5]. Arun Gangwar, Manushree Bhardwaj, An Overview: Peak to Average Power Ratio in OFDM system \& its Effect, International Journal of Communication and Computer Technologies Volume 01 - No.2, Issue: 02 September 2012 ISSN NUMBER : 2278-9723

[6]. Hong Xu1, Mustapha Benjillali2 and Leszek Szczeci ski, Bit error rate for rectangular QAM with arbitrary constellation mapping in Nakagami-m channels, Wireless communications and mobile computing Wireless. Commun. Mobile. Computing. 2008; 8:93-99 Published online 12 September 2006 in Wiley InterScience (www.interscience.wiley.com). DOI: 10.1002/wcm.437 